\title{
Bilateral Mastectomy as Radical Treatment of Gynecomastia Secondary to Antiretroviral Therapy in a Low-Income Setting: A Case Report
}

\author{
Mario Antunes ${ }^{1} \cdot$ Marcella Schiavone $^{2,3} \cdot$ Damiano $^{\text {Pizzol }}{ }^{3}(\mathbb{D}) \cdot$ Francesco Di Gennaro $^{4} \cdot$ \\ Rossana Ludovico ${ }^{5}$ Angela De Palma ${ }^{2}$
}

Published online: 11 May 2018

(c) The Author(s) 2018

\begin{abstract}
Gynecomastia is a common finding in males, with an incidence that varies widely globally. In $10-25 \%$ of cases, it is caused by drugs. Its pathophysiologic mechanism includes exposure to exogenous estrogens and medications that cause hypogonadism, antiandrogenic effects and hyperprolactinemia. Gynecomastia is associated with exposure to antiretroviral therapy (ART), particularly efavirenz. Sometimes surgery may be required as treatment. We report a case of a 46-year-old man receiving ART presenting with a marked bilateral breast enlargement who underwent bilateral mastectomy as the only successful treatment in a low-income setting.
\end{abstract}

Damiano Pizzol

d.pizzol@cuamm.org

1 Department of Surgery, Central Hospital of Beira, Beira, Mozambique

2 Department of Emergency and Organ Transplantation, Section of Thoracic Surgery, University of Bari "Aldo Moro", Bari, Italy

3 Operational Research Unit, Doctors with Africa CUAMM, Rua Fernao Mendes Pinto 165, Ponta Gea, 1363 Beira, Mozambique

4 Clinic of Infection Diseases, University of Bari "Aldo Moro", Bari, Italy

5 Plastic Surgery Consultant Emergency Department, Mater Dei Hospital, Bari, Italy

\section{Key Points}

One possible adverse drug effect of antiretroviral treatments, especially efavirenz, is male gynecomastia.

The development of prevention and early diagnosis strategies is crucial to improve treatment and patient health and to reduce health costs, especially in lowincome countries given the large number of patients with HIV in these countries.

Surgery can be a solution, especially in low-income countries facing late-stage disease and a lack of specialized health professionals and equipment.

\section{Introduction}

Gynecomastia is the enlargement of male breast tissue in men and is frequently observed in newborns, adolescents, and older men [1].

It should be differentiated from breast carcinoma and pseudogynecomastia, which is characterized by fat deposition without glandular proliferation.

Physiological gynecomastia, occurring in almost $25 \%$ of cases, is benign and self-limiting; this type is observed especially in young men, up to $65 \%$ of adolescents [2].

However, several conditions, such as the use of narcotic substances and drugs may induce proliferation of male breast tissue. Moreover, true gynecomastia is a common feature often related to estrogen excess, androgen deficiency, and a high elevation of sex hormone-binding globuline (SHBG) as a consequence of different endocrine 
disorders [3]. Non-endocrine illnesses, including liver failure, chronic kidney disease, and drug side effects [1], may also cause gynecomastia.

Drug-induced gynecomastia particularly merits deep consideration as it may account for as many as $25 \%$ of all cases of gynecomastia in adults. Although the mechanism is not fully clear, it may include estrogen-like activities, stimulation of testicular production of estrogens, inhibition of testosterone synthesis or blockade of androgen action. Anabolic steroids, particularly when used during the pubertal stage, may cause significant irreversible gynecomastia [4]. The pathophysiologic mechanism includes exposure to exogenous estrogens and medications that cause hypogonadism, antiandrogenic effects, and hyperprolactinemia [5].

Drugs definitely associated with the onset of gynecomastia are spironolactone, cimetidine, ketoconazole, human growth hormone (hGH), estrogens, human chorionic gonadotropin (hCG), antiandrogens, gonadotropinreleasing hormone $(\mathrm{GnRH})$ analogs, and $5-\alpha$ reductase inhibitors. Other drug classes seem to be protease inhibitors, and nucleoside reverse transcriptase inhibitors, although their association with gynecomastia is based on poor-quality evidence [6]. Finally, drugs probably associated with gynecomastia include risperidone, verapamil, nifedipine, omeprazole, alkylating agents, HIV therapies, anabolic steroids, alcohol, and opioids [7].

Efavirenz is the antiretroviral treatment (ART) most clearly associated with gynecomastia as a possible adverse drug reaction, especially with prolonged exposure [8].

We report a case of advanced-stage ART-related gynecomastia, treated with surgery and managed with satisfying results in a low-income setting.

\section{Case Report}

A 46-year-old man was admitted to Beira Central Hospital (Mozambique) with marked bilateral breast enlargement (Fig. 1). He was HIV positive, had been receiving ART (tenofivir/lamivudine/efavirenz $300 \mathrm{mg}$ ) for the past 5 years and reported gradual breast growth over the last year. Previous diseases were unremarkable, he was in good general condition, and his vital parameters were normal. Although he had lived for many months with the discomfort of this condition, he decided to treat the disease only after consultation with a traditional healer, and after it caused marked stigmata. He had no other signs and symptoms such as nipple discharge, bleeding, or breast pain.

We were unable to perform specific laboratory tests such as those for sex hormones and tumor markers, so, after clinical examination and excluding other main causes, such

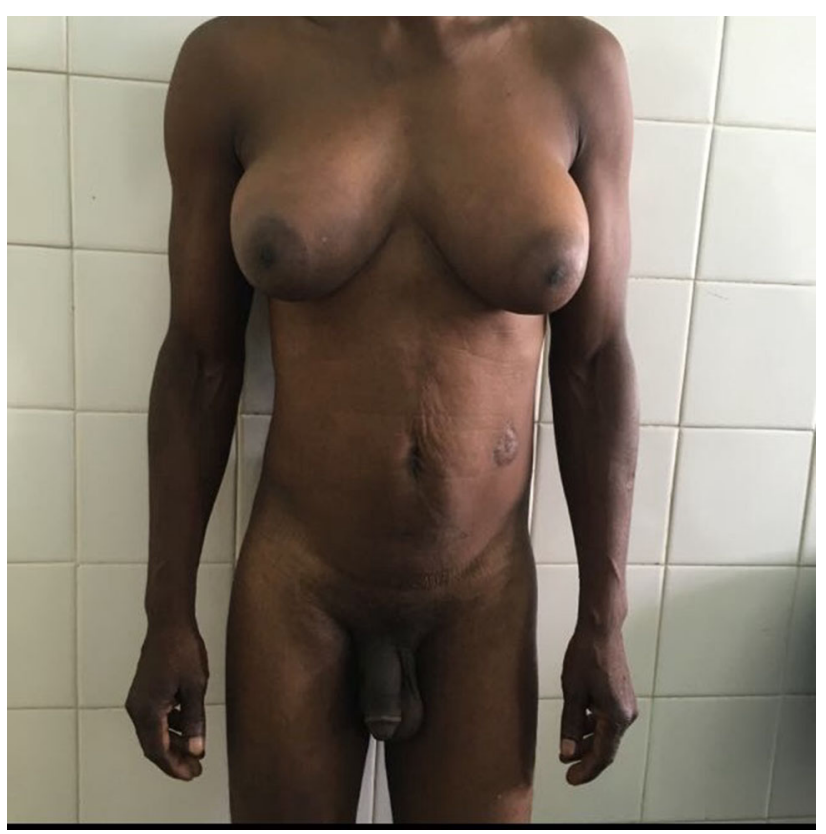

Fig. 1 46-year-old HIV-positive man with gynecomastia due to antiretroviral (ART) with tenofivir/lamivudine/efavirenz admitted to Beira Central Hospital (Mozambique) and successfully treated with bilateral mastectomy

as seminoma, liver diseases, and malnutrition, we clinically hypothesized the diagnosis of gynecomastia associated with ART.

Discontinuation of HIV therapy was not feasible, and the risk of recurrence with surgical undercorrection had to be avoided. Thus, a mastectomy, with double incision approach and nipple-areola complex (NAC) free transplantation, was proposed to the patient as radical treatment of his severe bilateral gynecomastia.

Pre-operative routine laboratory tests were normal: CD4 count $440 / \mathrm{mm}^{3}$.

The patient's chest characteristics and proportions were considered preoperatively, and the breasts were marked in a standing position: any side of lipodystrophy was noted in the patient's slim chest, no atypical thoracic conformation was detected, and severe bilateral hypertrophy with little asymmetry was noted in the context of a Simon III gynecomastia.

The NAC was removed as a full-thickness graft, trimmed to $2.5 \mathrm{~cm}$ and preserved in wet gauze until the end of the mastectomy. The mammary pocket was cleaned, and hemostasis was checked. The excess skin was removed, and the inframammary fold was closed in layers over a drain. Finally, the NAC was placed and fixed onto a deepithelialized bed previously marked symmetrically on the chest so the new nipple-sternal notch distance was $15 \mathrm{~cm}$ bilaterally.

The removed specimens weighed $1.5 \mathrm{~kg}$ (right) and $1.7 \mathrm{~kg}$ (left). 
Postoperative antibiotics and analgesics (amoxicillin and clavulanic acid $1000 \mathrm{mg}$ every $8 \mathrm{~h}$ and ibuprofen $600 \mathrm{mg}$ every $8 \mathrm{~h}$ ) were administered, and a moulage compressive dressing with non-stick gauze was maintained on the wounds for 3 days. The drain was then removed, and the patient was discharged on the fifth postoperative day without any complication. Compressive dressing of the wounds for 2 weeks was recommended. The patient continued with the same ART drugs and dosage. Although all instructions were given to the patient with a recommendation to return for follow-up, he never did so.

Histopathological examination confirmed the clinical diagnosis of gynecomastia, reporting epithelial and ductal cells and myxoid stroma.

\section{Discussion}

In low-income settings, it is common to be faced with extreme and late-stage diseases, with consequent management difficulties and negative outcomes. In fact, in these contexts, healthcare must deal with many sociocultural aspects such as superstitions and (often misinformed) traditional healers turning patients away from conventional medicine. Moreover, people are often reluctant to refer to conventional doctors because of the risk of stigma and marginalization in doing so.

We reported a case of gynecomastia in a patient receiving ART for HIV. The weak healthcare system, poorquality infrastructure and equipment, and limited tests and diagnostic procedures available to us meant an accurate diagnosis was impossible. However, given the clinical features and histopathological examination, we excluded other causes and, based on the literature, we concluded this case was ART related.

The management of this patient represented a challenge, most of all due to the delay before treatment, which led to physical, social, and psychological disability, reducing the chance of optimal results. For our patient, surgery represented the only therapeutic possibility, so we performed a bilateral mastectomy. We did not perform a reductive mastoplasty, even though it would have been aesthetically more suitable, because of the risk of glandular residue that with therapy would have again hypertrophied. The procedure we performed has already been reported in the literature as treatment for gynecomastia, with satisfactory results [9]. In this case, the mastectomy procedure was not difficult because of the well-delineated surgical plans and well-defined glandular tissue, which did not trespass into the pectoral regions or beyond the anterior axillary line.

The other big challenge in this case was the follow-up. In fact, the patient did not return for regular follow-up, which is common behavior in low-income settings, particularly when there are no complications. On the other hand, it was not possible to perform appropriate biochemical evaluation, including sex hormones, for adequate post-operative follow-up from an endocrine point of view.

\section{Conclusion}

Our approach to this case of gynecomastia was successful and effective, resulting in a satisfied patient, despite the extreme condition of the disease presentation. However, in low-income settings, it is crucial that the health system be strengthened in terms of both healthcare and prevention. In fact, if further studies confirm the frequent association between ART and gynecomastia, given the high prevalence of patients with HIV worldwide, it is crucial that prevention and early diagnosis strategies are developed to improve treatment and patient health and reduce health costs.

Acknowledgements The authors are very grateful to Dr. Pelizzon Alessandro of the School of Law and Justice, Southern Cross University, East Lismore, NSW 2480, Australia, for his kind and careful editing.

\section{Complaince with Ethical Standards}

Funding No financial support was received for the conduct of this study or preparation of this manuscript.

Conflict of interest Mario Antunes, Marcella Schiavone, Damiano Pizzol, Francesco Di Gennaro, Rossana Ludovico, and Angela De Palma have no conflicts of interest.

Informed consent The patient provided written informed consent to publish this case report and any accompanying images. A copy of the written consent may be requested for review from the corresponding author.

Open Access This article is distributed under the terms of the Creative Commons Attribution-NonCommercial 4.0 International License (http://creativecommons.org/licenses/by-nc/4.0/), which permits any noncommercial use, distribution, and reproduction in any medium, provided you give appropriate credit to the original author(s) and the source, provide a link to the Creative Commons license, and indicate if changes were made.

\section{References}

1. Sansone A, Romanelli F, Sansone M, Lenzi A, Di Luigi L. Gynecomastia and hormones. Endocrine. 2017;55(1):37-44.

2. Paris F, Gaspari L, Mbou F, Philibert P, Audran F, Morel Y, Biason-Lauber A, Sultan C. Endocrine and molecular investigations in a cohort of 25 adolescent males with prominent/persistent pubertal gynecomastia. Andrology. 2016;4(2):263-9.

3. Czajka-Oraniec I, Zgliczyński W. Phenotype of patients with gynecomastia. Endokrynol Pol. 2008;59(2):131-9.

4. Lo TE, Andal ZC, Lantion-Ang FL. Fluoxymesterone-induced gynaecomastia in a patient with childhood aplastic anaemia. BMJ Case Rep. 2015;6:2015. 
5. Eckman A, Dobs A. Drug-induced gynecomastia. Expert Opin Drug Saf. 2008;7(6):691-702.

6. Bowman JD, Kim H, Bustamante JJ. Drug-induced gynecomastia. Pharmacotherapy. 2012;32(12):1123-40.

7. Deepinder F, Braunstein GD. Drug-induced gynecomastia: an evidence-based review. Expert Opin Drug Saf. 2012;11(5):779-95.

8. Njuguna C, Swart A, Blockman M, Maartens G, Chisholm B, Stewart A, Uys A, Cohen K. Cases of antiretroviral-associated gynaecomastia reported to the National HIV \& Tuberculosis Health Care Worker Hotline in South Africa. AIDS Res Ther. 2016;13:40.

9. Muneer A, Laghari ZH, Shaikh AR, Laghari QA. Gynaecomastia: management in a developing country. J Ayub Med Coll Abbottabad. 2009;21(3):7-11. 